\title{
A New Implementation Method of the Reverse Engineering Algorithm to Elucidate Gene Functional Dependencies
}

\author{
Hidenori Inaoka Non-member (Tokyo Medical and Dental University) \\ Yutaka Fukuoka Non-member (Tokyo Medical and Dental University) \\ Akinori Ueno Member (Tokyo Denki University) \\ Isaac S Kohane Non-member (Harvard Medical School)
}

Keywords: reverse engineering algorithm, functional dependencies of genes, gene expression data, microarray

Various clustering methods are applied to characterizing gene expression data from DNA microarrays. However, to elucidate functional dependencies of genes analysis of gene associations is important. The REVerse Engineering ALgorithm (REVEAL) was developed for analyzing the functional dependencies. Although the algorithm has been tested using binary models of genetic networks, it remains unclear how the method or similar technology will operate with systems of continuous variables. In this study, the REVEAL was examined using noisy, continuous data. Then a new implementation method of REVEAL for such data was proposed. This implementation method was tested through simulations.

The general strategy of REVEAL is to use mutual information measures for extracting functional relationships between elements (genes) of a network using state transition tables. In the algorithm, the individual entropy for each gene, such as $H(X)$, the combined entropy, $H(X, Y)$ and the mutual information, $M(X, Y)$, of all pairs of genes are calculated first. Next, $M(X, Y) / H(X)$ is computed for all possible combinations. If $M(X, Y) / H(X)=1$, then $Y$ exactly determines $X$ and the functional relationship between $X$ and $Y$ can be determined based on the expression patterns of $X$ and $Y$. This process is repeated for all combinations of a gene and a pair of genes. If $M(X,[Y, Z]) / H(X)=1$, then the pair of $Y$ and $Z$ exactly determines $X$ and the relationship among the three can be determined in the same way. A simulation on application of the REVEAL to noisy, continuous data indicated that considerable refinements were required to apply the method to such data.

Then a new implementation method of REVEAL for noisy, continuous data was proposed. Generally, when comparing values $(\mathrm{A}, \mathrm{B})=(0.1,0.1)$ and $(\mathrm{A}, \mathrm{B})=(0.6,0.9)$, the latter pair suggests a stronger association between $\mathrm{A}$ and $\mathrm{B}$. The new method employed this idea. To weight values in gene expression data, we employed one threshold and multiple copies of binary data. In this method, for example, $(0.1$, $0.1)$ is converted into $(1,1)$ and $(0.6,0.9)$ is converted into six pairs of $(1,1)$ - i.e., the pair $(1,1)$ is copied six times. In
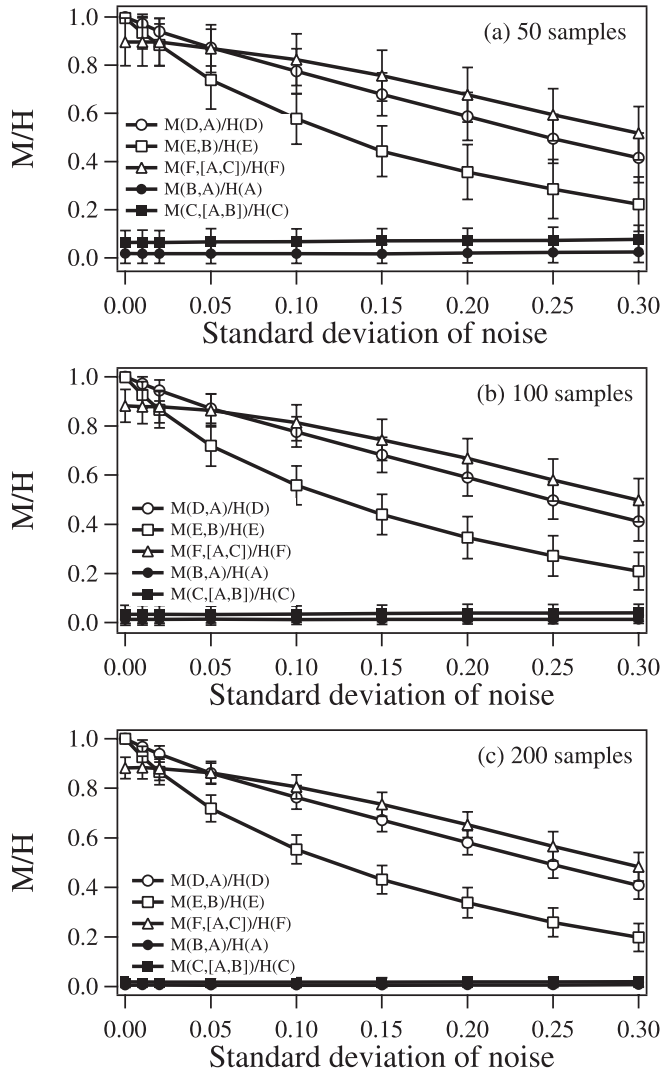

Fig. 1. Simulation results with the new implementation method of the REVEAL. This implementation method greatly improves the problem of low $\mathrm{M} / \mathrm{H}$ for large noise

addition to this, some implementation methods were investigated through numerical experiments. Figure 1 illustrates the result with the proposed implementation method, which was the best among the methods investigated. The results of the simulations demonstrated the potential of the proposed method for extracting gene associations. 


\title{
A New Implementation Method of the Reverse Engineering Algorithm to Elucidate Gene Functional Dependencies
}

\author{
Hidenori Inaoka* ${ }^{*}$ Non-member \\ Yutaka Fukuoka* ${ }^{* *}$ Non-member \\ Akinori Ueno*** Member $^{* * *}$ \\ Isaac S Kohane ${ }^{* * * *}$ Non-member
}

\begin{abstract}
Various clustering methods are applied to characterizing gene expression data from DNA microarrays. However, to elucidate functional dependencies of genes analysis of their associations is important. The REVerse Engineering ALgorithm (REVEAL) was developed for analyzing the functional dependencies. Although the algorithm has been tested using binary models of genetic networks, it remains unclear how the method or similar technology will operate with systems of continuous variables. In this study, first, the REVEAL was examined using noisy, continuous data and the results suggested that its application to such data required considerable refinement of the algorithm. Then a new implementation method of REVEAL was proposed. This implementation method was tested through numerical experiments. The results of the simulations demonstrated the potential of the proposed method for extracting gene associations.
\end{abstract}

Keywords: reverse engineering algorithm, functional dependencies of genes, gene expression data, microarray

\section{Introduction}

DNA microarray technology makes it possible to monitor expression patterns of thousands of genes ${ }^{(1)(2)}$. To characterize biological systems and/or disease states, such expression patterns are analyzed by various clustering methods: hierarchical clustering ${ }^{(3)}$, self-organized maps ${ }^{(4)}{ }^{(5)}$, and k-means clustering ${ }^{(6)}$. Among the most common, Eisen et $\mathrm{al}^{(3)}$ have demonstrated that a hierarchical clustering method is useful in the analysis of gene expression data. Also, Golub et al ${ }^{(5)}$ have shown that class discovery and class prediction by clustering expression data could be a generic approach to cancer classification. Most studies focus on the classification of genes and/or patients. However, to elucidate gene functional dependencies not only classification of gene expression data, but also analysis of gene associations is important.

Therefore the present study focuses on the analysis of functional relationships between/among genes from expression data. A systematic approach for modeling genetic networks has been also investigated $^{(7)-(9)}$. The S-system is a dynamic network model, which can infer

* Institute of Biomaterials and Biomedical Engineering, Tokyo Medical and Dental University,

2-3-10, Kanda-Surugadai, Chiyoda-ku, Tokyo 101-0062

** School of Biomedical Science, Tokyo Medical and Dental University,

1-5-45, Yushima, Bunkyo-ku, Tokyo 113-8510

*** Graduate School of Science and Engineering, Tokyo Denki University,

Ishizaka, Hatoyama-Cho, Hiki-Gun, Saitama 350-0394

**** Informatics Program, Childrens's Hospital, Harvard Medical School,

Boston, MA, USA a genetic network including groups of interdependent genes. The S-system belongs to a type of power-law formalism which includes a large number of parameters. Accordingly, to estimate these parameters, an immense amount of experimentally observed time-course data is required. Another approach for genetic network modeling is based on Boolean network. Liang et al. pro-

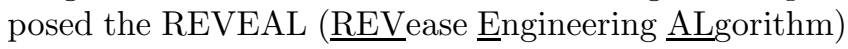
to infer functional relationships of genes from expression data. However, in the paper by Liang et al. ${ }^{(10)}$, they applied REVEAL only to binary models of genetic networks. It remains unclear how REVEAL or similar technologies will operate with systems of continuous variables.

In the present study, we propose a new implementation method of the REVEAL for noisy, continuous data. We begin this paper with a brief outline of REVEAL, followed by a description of the experimental procedures. Section 4 describes a new implementation method of REVEAL for noisy, continuous data.

\section{Reveal}

Liang et al. ${ }^{(10)}$ have proposed an algorithm called REVEAL for the inference of genetic network architectures. The general strategy of REVEAL is to use mutual information measures for extracting functional relationships between elements (genes) of a network using state transition tables, which are gene expression patters in functional genomics. The mutual information between $X$ and $Y, M(X, Y)$, corresponds to the remaining information of $X$ if we remove the information of $X$ that is not shared with $Y$ or vice versa. (See the following equations.) 


$$
M(X, Y)=H(X)-H(X \mid Y)=H(Y)-H(Y \mid X)
$$

where

$$
\begin{aligned}
& H(X)=-\sum_{i=0}^{l} p_{i} \log _{2} p_{i}, \\
& H(Y)=-\sum_{j=0}^{l} p_{j} \log _{2} p_{j}, \\
& H(X, Y)=-\sum_{i, j=0}^{l} p_{i j} \log _{2} p_{i j}, \text { and } \\
& H(Y \mid X)=H(X, Y)-H(X) .
\end{aligned}
$$

$H(X)$ is the entropy for $X$, which is a measure of the randomness in the expression pattern of $X$. Higher $H(X)$ means that the expression levels of $X$ are more randomly distributed. The logarithm used in the above equations, denoted as $\log _{2}$, is in base 2 . The probabilities, $p_{i}$ and $p_{j}(i, j=1$ or 0$)$, are calculated from the frequency of $X$ and $Y$ being 1 or 0 . Similarly, the joint probability, $p_{i j}(i, j=1$ or 0$)$, is determined by the frequency of $(X, Y)$ being $(0,0),(0,1),(1,0)$, or $(1,1)$.

In the algorithm, the individual entropy for each element, such as $H(X)$, the combined entropy, $H(X, Y)$ and the mutual information of all pairs of elements are calculated first. Next, $M(X, Y) / H(X)$ is computed for all possible combinations. If $M(X, Y) / H(X)=1$, then $Y$ exactly determines $X$ and the functional relationship between $X$ and $Y$ can be determined based on the expression patterns of $X$ and $Y$. This process is repeated for all combinations of an element and a pair of elements. If $M(X,[Y, Z]) / H(X)=1$, then the pair of $Y$ and $Z$ exactly determines $X$ and the relationship among the three can be determined in the same way.

\section{Simulations Using Binary Patterns}

In the paper by Liang et al. ${ }^{(10)}$, the REVEAL was examined using binary models of genetic networks without noise. However, the REVEAL should be examined using noisy data because real gene expression data are noisy. In this simulation, ten genes A, B, C, D, E, F, G, $\mathrm{H}$, I and $\mathrm{J}$, each of which had a sequence of $\mathrm{N}$ samples (corresponding to data from $\mathrm{N}$ simulated DNA chips), were used. We employed $\mathrm{N}=20,50,100$ and 200 to investigate the influence of the number of the samples. Six genes A, B, C, D, E and F were randomly given a value of 0 or 1 , while the other genes $\mathrm{G}, \mathrm{H}$, I and J were calculated according to Equations (2). Ten trials using different datasets were carried out.

$$
\begin{aligned}
& G=A \cdots \\
& H=\operatorname{not} B \\
& I=C a n d D \\
& J=E \operatorname{Eor} F
\end{aligned}
$$

In this simulation, about $5 \%$ of $\mathrm{N}$ samples were randomly chosen and inverted. More precisely, the number of inverted samples for each gene was 1, 2, 5 and 10
Table 1 . The number of gene associations correctly detected from the noisy binary patterns. Each number in the table shows the number of successful trials out of one hundred total

\begin{tabular}{|c|c|c|c|c|} 
& $\mathrm{G}=\mathrm{A}$ & $\mathrm{H}=\operatorname{not} \mathrm{B}$ & $\mathrm{I}=\mathrm{C}$ and $\mathrm{D}$ & $\mathrm{J}=\mathrm{E}$ or $\mathrm{F}$ \\
\hline $\mathrm{N}=20$ & 100 & 100 & 96 & 97 \\
$\mathrm{~N}=50$ & 100 & 100 & 100 & 100 \\
$\mathrm{~N}=100$ & 100 & 100 & 100 & 100 \\
$\mathrm{~N}=200$ & 100 & 100 & 100 & 100
\end{tabular}

Table 2. The number of false associations detected from noisy binary patterns. Each number in the table denotes the average number of false associations in a trial

$$
\left|\begin{array}{c|c}
\mathrm{N}=20 & 12.2 \\
\mathrm{~N}=50 & 6.9 \\
\mathrm{~N}=100 & 5.4 \\
\mathrm{~N}=200 & 3.4
\end{array}\right|
$$

respectively for $\mathrm{N}=20,50,100$ and 200 . We generated one hundred datasets by adding ten different noise realizations to each of ten datasets. In this situation, the equations, $M(X, Y) / H(X)=1$ and $M(X,[Y, Z]) / H(X)$ $=1$, are no longer true even if $X$ is a function of $Y$ or $[Y, Z]$.

However, one can expect that $M(X, Y) / H(X)$ and $M(X,[Y, Z]) / H(X)$ are relatively large values when $X$ is a function of $Y$, and $X$ is a function of $[Y, Z]$, respectively. Accordingly, in the simulations, a functional association was determined in the following way. First, we calculated the average over all possible combination of $M(X, Y) / H(X)$ and $M(X,[Y, Z]) / H(X)$. Then the standard deviation of all $M / H$ was calculated. If $M(X, Y) / H(X)$ or $M(X,[Y, Z])$ was greater than the sum of the average and the standard deviation, we defined that $X$ was a function of $Y$ or $X$ was a function of $[Y, Z]$.

As shown in Table 1, the REVEAL could capture all four relationships almost perfectly. It failed to capture the associations of the triplets in three or four trials (out of one hundred total) when $\mathrm{N}=20$. Table 2 summarizes the number of false associations. The numbers in the table denote the average number of false associations over one hundred trials. The REVEAL detected many false associations. In particular, when $\mathrm{N}=20$, on average 12.2 false associations were detected in a trial. As $\mathrm{N}$ increased, the average number decreased rapidly. For example, it became almost half for $\mathrm{N}=50$. However, even for $\mathrm{N}=200$, the method may capture three or more false associations. This is a weak point of the REVEAL and thus its application to noisy data requires considerable refinements of the method to reduce the amount of false detection. We will address this problem using noisy, continuous data in the next section.

\section{Implementation of REVEAL for Noisy, Continuous Data}

Real gene expression data have continuous values, although in the previous section, the REVEAL was examined using binary patterns. In this section, we investigated implementation methods of the REVEAL for noisy, continuous data. 


\subsection{A Simple Implementation Method A}

straightforward way of adapting REVEAL to noisy data would be to use a threshold to convert continuous data into a binary pattern. To examine this implementation method, the following simulations were carried out. Six genes A, B, C, D, E and F, each of which had N samples $(\mathrm{N}=50,100$, or 200$)$, were used. Each sample of the genes $\mathrm{A}, \mathrm{B}$ and $\mathrm{C}$ was given a value taken from a uniform distribution between -1 and 1 while the genes $\mathrm{D}, \mathrm{E}$ and $\mathrm{F}$ were calculated according to the following equations.

$$
\begin{aligned}
& D=0.5 A \ldots \ldots \ldots \ldots \ldots \ldots \ldots \ldots \ldots \ldots \ldots \ldots \\
& E=-0.3 B \\
& F=-0.2 A+0.7 C
\end{aligned}
$$

The simulations were performed with the following levels of standard deviation of noise: $0,0.05,0.1,0.15$, $0.2,0.25$ and 0.3 . One hundred runs were obtained for each noise level. In each run, the values of $\mathrm{A}, \mathrm{B}$ and $\mathrm{C}$ were randomized and a different zero-mean Gaussian noise realization was added to the values in all genes. Before applying REVEAL, the data were converted into a binary pattern using a threshold of 0 .

Figure 1 depicts the experimental results: (a) $\mathrm{N}=50$, (b) $\mathrm{N}=100$ and (c) $\mathrm{N}=200$. The horizontal axis indicates the standard deviation of the noise while the vertical axis shows the value of $M(D, A) / H(D)$ and so on. The data shown in each graph can be divided into two groups. The first group consists of $M(D, A) / H(D)$, $M(E, B) / H(E)$ and $M(F,[A, C]) / H(C)$. Each of these gene combinations is defined in Equations (3), and consequently, $M / H$ for these combinations should be a large value. The other group includes $M(B, A) / H(A)$ and $M(C,[A, B]) / H(C)$. Because the genes $\mathrm{A}, \mathrm{B}$ and $\mathrm{C}$ were independently given a random number between -1 and $1, M / H$ in this group should be close to zero.

As shown in Fig. 1, with low-amplitude noise (the standard deviation $<0.2$ ), the two groups were clearly separated. Both $M(B, A) / H(A)$ and $M(C,[A, B]) / H(C)$ remained nearly zero for all noise amplitudes. In contrast, regardless of $\mathrm{N}, M / H$ in the first group became smaller as the amplitude of noise increased. In particular, $M(E, B) / H(E)$ was almost the same as $M(B, A) / H(A)$ and $M(C,[A, B]) / H(C)$ with a standard deviation of 0.3 . The number of samples, $\mathrm{N}$, had no obvious effect on the average $M / H$, but the standard deviation of $M / H$ became smaller as $\mathrm{N}$ increased. These results suggest that this implementation method does not work well for very noisy data. It should be noted that $M(F,[A, C]) / H(F)$ was not 1 even if no noise was introduced. This phenomenon is explained as follows. The sign of $\mathrm{F}$ was determined depending on the values of $\mathrm{A}$ and $\mathrm{C}$; if $\mathrm{A}<3.5 \mathrm{C}$, the sign of $\mathrm{F}$ was same as that of $\mathrm{C}$, otherwise it was opposite of A. Thus, there was no obvious logical relationship among the resultant binary patterns.

4.2 A New Implementation Method Generally, when comparing values $(\mathrm{A}, \mathrm{B})=(0.1,0.1)$ and $(\mathrm{A}, \mathrm{B})=$ $(0.6,0.9)$, the latter pair suggests a stronger association
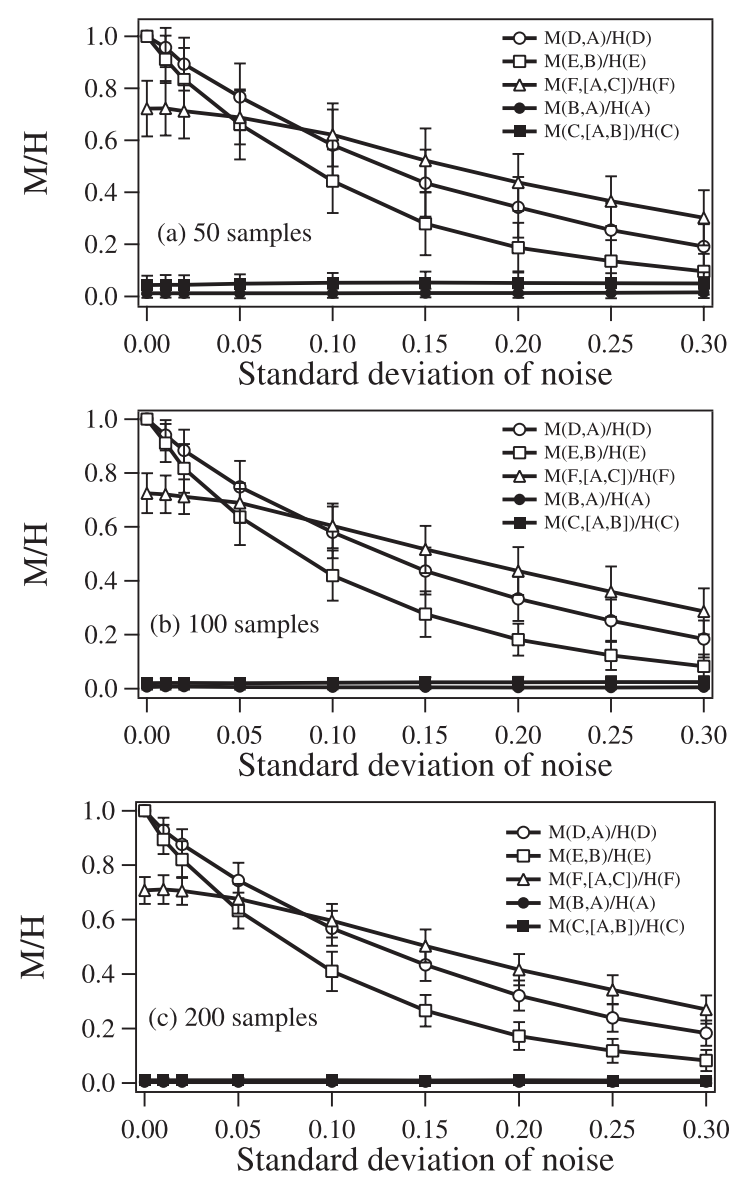

Fig. 1. Simulation results with the simple implementation method of the REVEAL. The results suggest that this implementation method does not work well for very noisy data

between $\mathrm{A}$ and $\mathrm{B}$. However, in the method described in 4.1, values 0.1 and 0.9 had the same effect. That is, both pairs were converted into $(1,1)$ before calculating entropy. It is expected that improving this situation would make the REVEAL more robust against noise. REVEAL is applicable to data having three or more levels (or discretized: "bins") although it has so far been investigated solely using binary data (i.e., 0 or 1 ).

A simple method to weight values in gene expression data is discretizing the data using multiple bins. Butte and Kohane ${ }^{(11)}$ developed such a method and applied it to a publicly available gene expression dataset. They calculated the range of values for each gene and then divided the range into $n$ sub-ranges (bins). In their method, entropy is defined by

$$
H(X)=-\sum_{i=1}^{n} p_{i} \log _{2} p_{i}
$$

Here, the probability, $p_{i}$, is calculated from the frequency of $X$ being in the $i$-th bin. Obviously, with $\mathrm{n}$ $=2$, this method is the same as the one in 4.1. In the paper by Butte and Kohane ${ }^{(11)}$, they used $n=10$. In the case of $\mathrm{n}=10$, the data used in 4.1 are converted into ten levels using multiple thresholds of $-0.8,-0.6,-0.4$ and so on. This operation yields one hundred combinations of levels between A and B (compared to four combina- 
tions for binary data). Accordingly, this method could be computationally expensive. In what follows, we refer to this method as the multiple-bins method.

Instead, we employed one threshold and multiple copies of binary data. In this method, for example, $(0.1$, $0.1)$ is converted into $(1,1)$ and $(0.6,0.9)$ is converted into six pairs of $(1,1)$ - i.e., the pair $(1,1)$ is copied six times. In this way, values in gene expression data can be weighted. The number of copies can be arbitrarily determined. In the present study, we determined the number of copies, c, by

$$
c=\min (|X|,|Y|) / s \cdots \ldots \ldots \ldots \ldots \ldots \ldots \ldots \ldots \ldots \ldots
$$

where $|X|$ and $|Y|$ denote the absolute values of $X$ and $Y$, respectively. A small positive number such as 0.1 is used as $s$. If the term on the right-hand side becomes $0, c$ is set to 1 . Conversely, if $c$ exceeds a predetermined maximum value, $c_{\max }$, it is set to $c_{\max }$. Accordingly, if $s=1$ and the maximum value of $|X|$ and $|Y|$ is 1 , this implementation method equals the previous method in 4.1. Hereafter, this method is called the multiple-copies method.

In order to compare the two implementation methods of the REVEAL, we carried out simulations using the same data as 4.1. In the simulations, $\mathrm{n}$ was set to 10 for the multiple-bins method, while in the multiple-copies method, we employed $s=0.1$ and $c_{\max }=10$. The other conditions, such as noise levels and the number of samples, were identical to the previous set of simulations. In addition, we compared the CPU time required to calculate $M / H$ for 162,000 triplets. This approximately corresponds to the total number of all possible triplets out of 100 genes. A Pentium IV processor $(1.4 \mathrm{GHz})$ was used for the calculation.

Figure 2 illustrates the results with the multiple-bins method: (a) $\mathrm{N}=50$, (b) $\mathrm{N}=100$ and (c) $\mathrm{N}=200$. In each graph, the value of $M / H$ (such as $M(D, A) / H(D)$ ) was plotted against the standard deviation of the noise. There were two groups of plots. The first group consisted of $M(D, A) / H(D), M(E, B) / H(E)$ and $M(F,[A, C]) / H(C)$. Each of these combinations was defined in Equations (3), and consequently, $\mathrm{M} / \mathrm{H}$ for these combinations should be a large value. The other group included $M(B, A) / H(A)$ and $M(C,[A, B]) / H(C)$. The genes $\mathrm{A}, \mathrm{B}$ and $\mathrm{C}$ were independently assigned a random number between -1 and 1 . In other words, there was no functional relationship among these genes.

As shown in Fig. 2, the values of $M / H$ in the first group decreased as the standard deviation of noise increased, whereas $M / H$ for the second group was constant. These are similar to what we observed in Fig. 1. However, $M(F,[A, C]) / H(C)$ was almost constant in Fig. 2(a) and the values of $M / H$ for the second group, especially when $\mathrm{N}=50$, were much larger than those in Fig. 1. In addition, as noise became larger, $M / H$ for the pairs and triplet in the first group rapidly decreased and converged on $M(B, A) / H(A)$ and $M(C,[A, B]) / H(C)$, respectively. This behavior of $M / H$ was very different from that in Fig. 1.

Figure 3 depicts the results with the multiple-copies
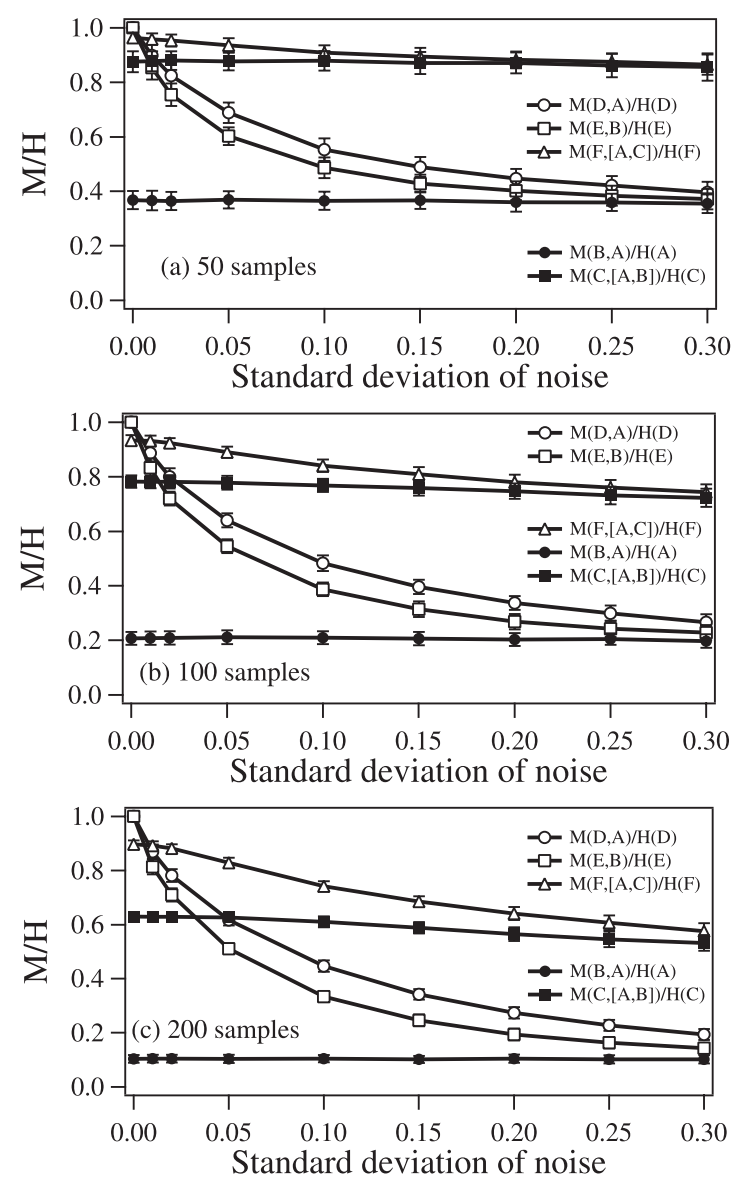

Fig. 2. Simulation results with the multiple-bins implementation of the REVEAL

method: (a) N=50, (b) N=100 and (c) N=200. The horizontal and vertical axes are the same as Fig. 2. As shown in Fig. 3, this implementation method greatly improves the problem of low $M / H$ for large noise in the simple method. For almost all noise levels $M / H$ was greater than 0.5 except $M(E, B) / H(E)$. The reason for the low $M(E, B) / H(E)$ lay in small absolute values of gene E (see Eqs. (3)), and thus data of E were easily affected by noise to the extent where data bits were easily inverted. However, this problem would be resolved by data normalization. Similar to the simple implementation method, $\mathrm{N}$ had an effect only on the standard deviation of $M / H$. With $\mathrm{N}=100$ or 200 , the standard deviations became small to the extent that the two groups were divided clearly for all noise levels.

4.3 Evaluation of the New Implementation Method Using Real Data The robustness of the proposed implementation method has been verified using real data. We used microarray expression data from Escherichia coli as real data (12 samples, 4290 genes) ${ }^{(12)}$. Calculating mutual information for all possible triplets from the 4290 genes requires enormous computational time. Therefore the dataset was divided into small subsets. First, the information about all $E$. coli pathways was obtained from the database of $\mathrm{Ky}-$ oto Encyclopedia of Genes and Genomics (KEGG) ${ }^{(13)}$, and then the expression dataset was divided into subsets based on the genes included in each pathway. Each 

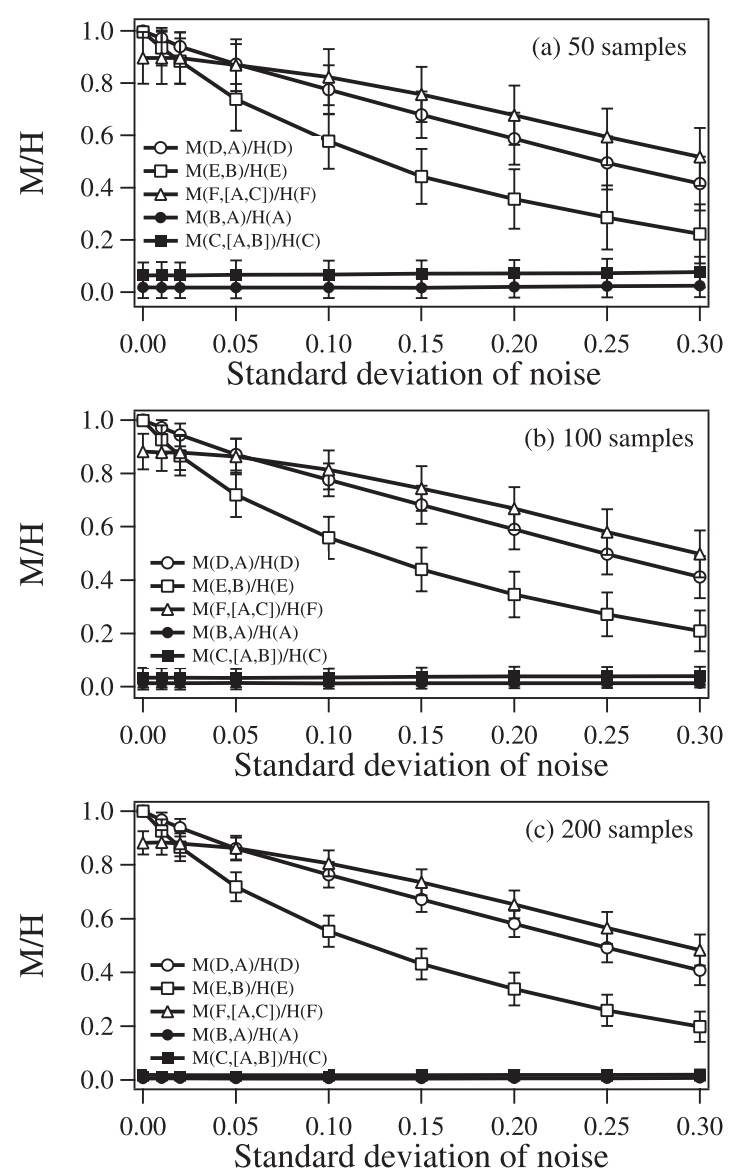

Fig. 3. Simulation results with the new implementation method of the REVEAL. This implementation method greatly improves the problem of low $\mathrm{M} / \mathrm{H}$ for large noise

Table 3. Triplets detected from the real data using the proposed method

\begin{tabular}{|l|l|l|} 
& genes in triplet & pathway \\
\hline 1 & folD, glyA, metF & One carbon pool by folate \\
2 & tesB, yjhQ, paaF & Limonene and pinene degradation \\
3 & adhC, yiaY, ydfG & Bile acid biosynthesispath \\
4 & adhC, yiaY, gst & Metabolism of xenobiotics by cytochrome P450
\end{tabular}

subset had about 23 genes in average. In this study, we focused on triplets because functional relationships of pairs can be easily detected by other methods. By applying the proposed method to each subset, a few triplets which have large $M / H$ was detected. However, most of the triplets included correlated pairs, and accordingly, $M / H$ of the triplets became relatively large. Such triplets were excluded. Table 3 shows the results.

\section{Discussion}

5.1 Merits and Problems of the REVEAL The simulation results in Section 3 revealed the merits and problems of REVEAL. The greatest merit of the REVEAL is that it can be applicable to an analysis of triplets. However, for noisy data, the method detects several false associations. With $\mathrm{N}=20$, it detected a considerable amount of such associations although it captured all the true relationships. It should be noted that spurious associations can be ruled out through bi-
Table 4. The results obtained from the dataset shown in Fig. 4 with the multiple-copies method. Parameters, s of 0.1 and 0.05 , and $c_{\max }$ of 10 and 20 were examined

\begin{tabular}{|c|c|c|c|} 
& $\mathrm{s}=0.1, c_{\max }=10$ & $\mathrm{~s}=0.05, c_{\max }=10$ & $\mathrm{~s}=0.05, c_{\max }=20$ \\
\hline$M(X, Y) / H(X)$ & 0.10 & 0.08 & 0.12 \\
$M(X, Z) / H(X)$ & 0.91 & 0.94 & 0.95
\end{tabular}

ological validation.

Although we tested only one threshold of $M / H$, the threshold also affects the number of false associations. If the threshold is too small, REVEAL detects a considerable number of false relationships. In contrast, with too large a threshold, the method may miss some of the true associations. However, because of the validation procedure, a slightly smaller threshold may be acceptable. Thus, exploring a range of thresholds would be a next step as would the determination of a threshold through the permutation method proposed by Butte and Kohane ${ }^{(11)}$.

5.2 Comparison of the Multiple-Bins and Multiple-Copies Methods When comparing Figs. 2 and $3, M / H$ with the multiple-bins method was large even for the unrelated pairs/triplets (such as $M(C,[A, B]) / H(C))$. This phenomenon could be explained by the following facts.

In the multiple-bins method, the number of bins, $n$, has significant effect on the quality of the result. When $n$ becomes larger, the size of bins becomes smaller. Thus the probability that two or more data enter into the same bin becomes small. Figure 4 schematically explains this using two datasets ( $\mathrm{X}$ and $\mathrm{Y}, \mathrm{X}$ and $\mathrm{Z}$ ); there was no relationship between $\mathrm{X}$ and $\mathrm{Y}$ whereas $\mathrm{Z}$ strongly correlated with $\mathrm{X}$. Although the distributions of the data were markedly different between the two scatter plots, only one or two bins include two data (shown in grey bin). Therefore the value of $H(X, Y)$ close to $H(X, Z)$, and then $M / H$ becomes relatively large $(\mathrm{n}=10: H(X, Y)=4.1, H(X, Z)=3.6$, $M(X, Y) / H(X)=0.54, M(X, Z) / H(X)=0.83 . \mathrm{n}=$ $20: H(X, Y)=4.2, H(X, Z)=4.1, M(X, Y) / H(X)=$ $0.72, M(X, Z) / H(X)=0.89)$.

In the multiple-copies method, the parameter that corresponds to $\mathrm{n}$ is the number of copies, c. In order to investigate the effect of $\mathrm{c}$ on the quality of the result, we analyzed the dataset shown in Fig. 4 with $\mathrm{s}=0.1$ and 0.05. (Remember $\mathrm{c}$ is determined by s; see Eq. (5).) In the case of $\mathrm{s}=0.05$, both $c_{\max }=10$ and 20 were examined. The results given in Table 4 indicate that the multiple-copies method works well in all cases. Unlike $\mathrm{n}$ in the multiple-bins method, the quality of the result is well preserved for either s in the multiple-copies method.

The influence of noise is small in the multiple-copies method. Because the sign of large absolute values is hardly changed by additive noise. The multiple-copies method employs only one threshold of zero and weights a pair/triplet according to the absolute values of samples. Hence, the method is more robust against noise.

5.3 Result of Real Data Analysis As shown in Table 3, only four triplets are detected. Triplet 1) 


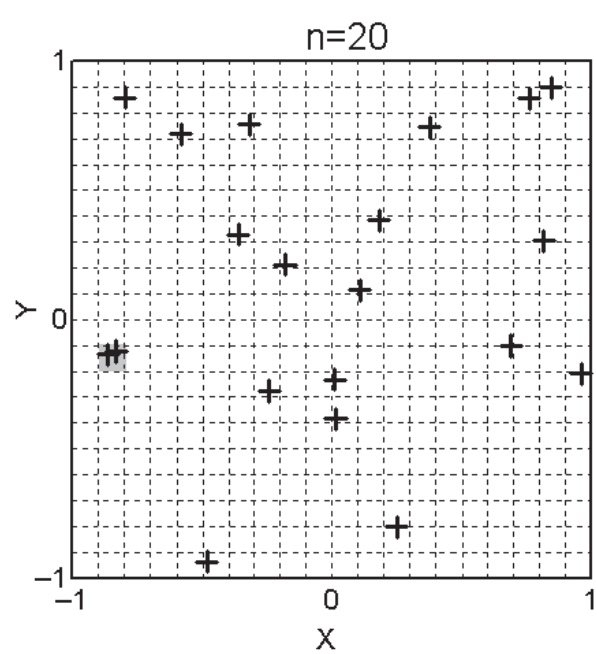

$H(X)=3.8 \quad H(Y)=3.1 \quad H(X, Y)=4.2$

$M(X, Y)=2.7 \quad M(X, Y) / H(X)=0.72$

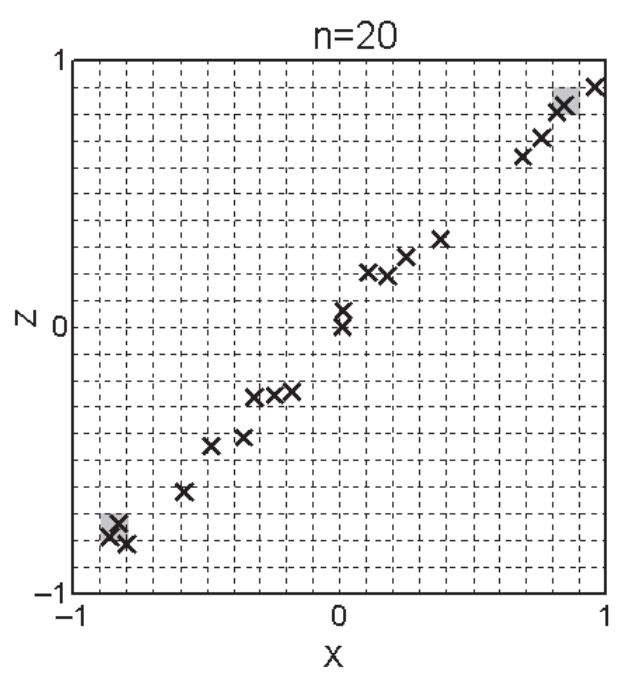

$H(X)=3.8 \quad H(Z)=3.7 \quad H(X, Z)=4.1$

$M(X, Z)=3.4 \quad M(X, Z) / H(X)=0.89$

Fig. 4. A schematic explanation for large $M / H$ by the multiple-bins method. The results using 20 bins are shown. There was no relationship between $X$ and $Y$ in upper, whereas $Z$ was calculated by adding a random small value to $X$. Therefore, $Z$ strongly correlated with $X$. Despite the different relationships between $\mathrm{X}$ and $\mathrm{Y}, \mathrm{X}$ and $\mathrm{X}$, the $M / H$ values are relatively large for both pairs

is deemed to be biologically meaningful because the enzymes which are coded with the three genes are linked by 5,10 -Methylene-THF on the pathway map ('one carbon pool by folate'). The functional relationship of the triplet is best described with $($ glyA $)=($ folD $)$ AND (metF). This example demonstrates that the proposed method can detect the functional relationship of genes which are directly connected in a pathway. The genes involved in Triplets 2), 3) and 4) in Table 3 have a few enzymes or compaunds between them, and accordingly, the functional relationships among these triplets are currently not supported by the structures of the pathways. Verifying these results through biological experiments is important future work. The microarray data used in this analysis includes only 12 arrays, therefore it is unlikely that the reliability of the result is very high. To obtain a more reliable result, a larger data set is desirable.

\section{Conclusion}

In the present study, we focused on the analysis of functional relationships between/among genes from expression data. First, we examined a reverse engineering algorithm using binary patterns. The experimental results suggested that application of the REVEAL for noisy data required considerable refinement of the reverse engineering methodology. Then we applied REVEAL to noisy, continuous data and proposed a new implementation method of REVEAL for such data. We carried out simulations to compare the implementation method with a similar method reported previously. The results demonstrated the potential of the proposed method.

(Manuscript received Jan. 22, 2007, revised May 18, 2007)

\section{References}

(1) D.J. Lockhart, H. Dong, M.C. Byrne, M.T. Follettie, M.V. Gallo, M.S. Chee, M. Mittmann, C. Wang, M. Kobayashi, H. Horton, and E.L. Brown: "Expression monitoring by hybridization to high-density oligonucleotide arrays", Nature Biotechnol., Vol.14, pp.1675-1680 (1996)

( 2 ) J. DeRisi, L. Penland, P.O. Brown, M.L. Bittner, P.S. Meltzer, M. Ray, Y. Chen, Y.A. Su, and J.M. Trent: "Use of a cDNA microarray to analyse gene expression patterns in human cancer", Nature Genet., Vol.14, pp.457-460 (1996)

( 3 ) M. Eisen, P.T. Spellman, P.O. Brown, and D. Botstein: "Cluster analysis and display of genome-wide expression patterns", Proc. Natl. Acad. Sci. USA, Vol.95, pp.14863-14868 (1998)

(4) P. Tamayo, D. Slonim, J. Mesirov, Q. Zhu, S. Kitareewan, E. Dmitrovsky, E.S. Lander, and T.R. Golub: "Interpreting patterns of gene expression with self-organizing maps: Methods and application to hematopoietic differentiation", Proc. Natl. Acad. Sci. USA, Vol.96, pp.2907-2912 (1999)

( 5 ) T.R. Golub, D.K. Slonim, P. Tamayo, C. Huard, M. Gaasenbeek, J.P. Mesirov, H. Coller, M.L. Loh, J.R. Downing, M.A. Caligiuri, C.D. Bloomfield, and E.S. Lander: "Molecular classification of cancer: class discovery and class prediction by gene expression monitoring", Science, Vol.286, pp.531-537 (1999)

(6) S. Tavazoie, J.D. Hughes, M.J. Campbell, R.J. Cho, and G.M. Church: "Systematic determination of genetic network architecture", Nature Genet., Vol.22, pp.281-285 (1999)

( 7 ) T. Akutsu, S. Miyano, and S. Kuhara: "Inferring qualitative relations in genetic networks and metabolic pathways, Bioinformatics, Vol.16(8), pp.727-734 (2000)

(8) S. Kikuchi, D. Tominaga, M. Arita, K. Takahashi, and M. Tomita: "Dynamic modeling of genetic networks using genetic algorithm and S-system", Bioinformatics, Vol.19(5), pp.643650 (2003)

(9) T. Hanai, H. Hamada, and M. Okamoto: "Application of bioinformatics for DNA microarray data to biocsience, bioengineering and medical fields", J. Biosci. Bioeng., Vol.101(5), pp.377-384 (2006)

(10) S. Liang, S. Fuhrman, and R. Somogyi: "REVEAL, a general reverse engineering algorithm for inference of genetic network architectures", Pacific Symp. Biocomp., Vol.3, pp.18-29 (1998)

(11) A.J. Butte and I.S. Kohane: "Mutual information relevance networks: functional genomic clustering using pairwise entropy measurements", Pacific Symp. Biocomp., Vol.5, pp.418429 (2000) 
(12) C. Bianco, E. Imperlini, R. Calogero, B. Senatore, P. Pucci, and R. Defez: "Indole-3-acetic acid regulates the central metabolic pathways in Escherichia coli", Microbiology, Aug;152(Pt 8), pp.2421-2431 (2006)

(13) M. Kanehisa, S. Goto, M. Hattori, K.F. Aoki-Kinoshita, M. Itoh, S. Kawashima, T. Katayama, M. Araki, and M. Hirakawa: "From genomics to chemical genomics: new developments in KEGG", Nucleic Acids Res., Vol.34, pp.D354D357 (2006)

Hidenori Inaoka (Non-member) received the Ph.D. degree

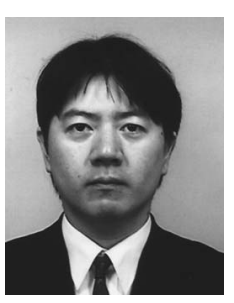
in medical science from Tokyo Medical and Dental University, Tokyo, Japan, in 2003. He is currently a Research Associate of Institute of Biomaterials and Bioengineering, Tokyo Medical and Dental University, Tokyo, Japan. His research interests include bioinformatics and biosystems modeling.

Yutaka Fukuoka (Non-member) received the Ph.D. degree

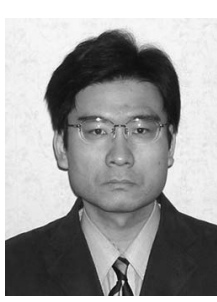
in electrical engineering from Keio University, Yokohama, Japan, in 1992. He was a Visiting Scientist at Harvard-MIT Division of Health Sciences and Technology, Cambridge, MA, in 1999 and 2000. He is currently an Associate Professor at School of Biomedical Science, Tokyo Medical and Dental University, Tokyo, Japan. His research interests include bioinformatics and biosystems modeling.
Akinori Ueno (Member) received the B.S. degree in elec-

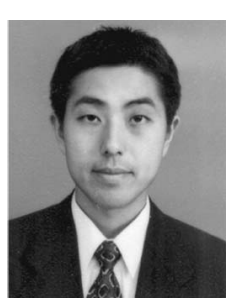
trical engineering from Keio University, Yokohama, Japan, in 1994, and the M.S. and Ph.D. degrees in biomedical engineering from Keio University, Yokohama, Japan, in 1996 and 1999, respectively. In 1999, he joined the faculty of the Electronic and Computer Engineering Department, Tokyo Denki University and has been an Associate Professor since 2004. His research interests include biomedical instrumentation and intelligent human-machine interfaces. Dr. Ueno is the recipient of several research awards from the Society of Instrument and Control Engineers, the Japan Society of Medical Electronics and Biological Engineering, and the Society of Life Support Technology.

Isaac S Kohane (Non-member) is the director of the Children' s Hospital Informatics Program and is the Henderson Associate Professor Health Sciences and Technology at Harvard Medical School. He leads multiple collaborations at Harvard Medical School and its hospital affiliates in the elucidation of regulatory networks of genes and the interaction between genotype and phenotype using a variety of bioinformatics techniques. His research builds on his doctoral work in computer science on decision support and subsequent research in machine learning applied to biomedicine. He has published over 100 papers in biomedical informatics. He is a Fellow of the American College of Medical Informatics and a Fellow of the Society for Pediatric Research. Dr. Kohane is also a practicing pediatric endocrinologist at Children ' s Hospital in Boston. 